\title{
Coffee plant image segmentation and disease detection using JSEG algorithm
}

\author{
Jeferson de Souza Dias, José Hiroki Saito, \\ Centro Universitário Campo Limpo Paulista \\ Campo Limpo Paulista, Brasil \\ jsdiasp@gmail.com
}

\begin{abstract}
Brazil is the largest coffee producer in the world, and then there are many challenges to maintain the high quality and purity of the beans. Thus, it is important to study coffee plants, and help agronomists to detect diseases, such as rust, with resources of computer science. In this work, it is described experiments using image segmentation algorithm JSEG, which is capable to segment images in multi-scale. Using a coffee tree image database RoCoLe (Robusta Coffee Leaf Images), the JSEG algorithm is used to segment these images in four scales. It is selected typical segments in each scale and they are grouped using similarity of normalized color histograms. In this way the several scales segmentations are compared. It is concluded that the segments in scales 1 and 2, in which the colors are more homogeneous then in scales 3 and 4, are adequate to use as training samples for the detection of rust diseases.
\end{abstract}

Keywords: $J S E G$, image segmentation, multi-scale, coffee diseases, rust, clustering.

\section{INTRODUÇÃ̃o}

Trabalhos sobre detecção de doenças em plantas, incluindo cafeeiros, utilizando técnicas computacionais tem sido desenvolvido ao longo dos anos, sendo uma dessas doenças conhecida como ferrugem ou ferrugem alaranjada, causada pelo fungo Hemileia vastatrix [1]. Suhartono et al. [2] descreveram sobre o desenvolvimento de um aplicativo inteligente para detecção de doenças em cafeeiros. O método usado é a lógica nebulosa e árvore de decisão, usando classificação hierárquica. Barbedo [3] fez uma revisão sobre os principais desafios na identificação automática de doenças em plantas, baseada em imagens visíveis, desenvolvido na Embrapa Informática Agrícola, sediada em Campinas, São Paulo. Segundo o autor, as técnicas propostas na detecção de doenças em imagens de plantas são limitadas na captura dessas imagens, pela existência de fundos de imagem que não podem ser separadas facilmente da região de interesse, normalmente, folhas e troncos. $\mathrm{O}$ artigo provê uma análise dos desafios para os problemas encontrados, as causas e as soluções encontradas. Os problemas encontrados são a falta de fronteiras claras em torno dos sintomas, variação nas condições de captura, doenças que produzem sintomas variáveis, sintomas produzidos por diferentes doenças que se manifestam simultaneamente e diferentes doenças que produzem sintomas semelhantes. A conclusão é que a aplicação do processamento digital de imagens e da visão computacional no diagnóstico de plantas ainda é nova, e existem muitas alternativas a serem exploradas para minimizar os problemas apontados. Com mais poder computacional, estratégias antes proibitivas tornam-se cada vez mais viáveis e a melhoria na tecnologia de imageamento, resultando em imagens mais confiáveis, permite o desenvolvimento de ferramentas mais precisas e poderosas.

Um trabalho importante sobre classificação de folhas de plantas, baseadas em textura foi descrito por Metre e Ghorpade [4]. Mengistu et al. [5] descreveram pesquisas sobre folhas cafeeiras da Etiópia, para o diagnóstico de doenças, usando técnicas de Redes Neurais Artificiais e classificação em $\mathrm{k}$ vizinhos mais próximos ( $k$-Nearest Neighbors - KNN), mapas auto-organizáveis (Self Organizing Map - SOM), e funções de base radial. Para a segmentação utilizaram técnicas como Otsu, Fuzzy C-Means, $k$-means e Distribuição Gaussiana e combinações de $k$-means e Distribuição Gaussiana. Para a segmentação, a conclusão foi que essa técnica combinada foi melhor que as demais técnicas. Em geral, o fator de coloração se mostrou mais representativo que a textura. Mettleq e Abu-Naser [6] descreveram um sistema baseado em regras para diagnóstico de doenças cafeeiras, utilizando a linguagem CLIPS ( $C$ Language Integrated Production System), desenvolvido pela NASA. O sistema desenvolvido inclui nove tipos de doenças no cafeeiro. Parraga-Alava [7] descreveram sobre uma base de dados denominada RoCoLe, (Robusta Coffee Leaf images dataset) sobre imagens de folhas cafeeiras. $\mathrm{O}$ banco de dados contém 1560 imagens de folhas com ácaros vermelhos e manchas de ferrugem. Além disso, o conjunto de dados inclui anotações sobre objetos (folhas), estado (saudável e não saudável) e a gravidade da doença (área da folha com manchas). As imagens foram todas obtidas em condições do mundo real no mesmo campo de cafeeiros usando uma câmera de smartphone. O conjunto de dados RoCoLe facilita a avaliação do desempenho de algoritmos de aprendizado de máquina usados na segmentação de imagens e em problemas de classificação relacionados ao reconhecimento de doenças de plantas. O conjunto de dados está disponível gratuita e publicamente, o que motivou o seu uso neste trabalho.

Sorte [9] desenvolveram um trabalho sobre o reconhecimento de doenças em folhas cafeeiras, utilizando técnicas de Deep Learning e atributos de textura. Foram considerados dois tipos de atributos de textura: estatísticos e padrões binários locais, para a classificação, utilizando uma rede neural multicamadas, para verificar a acurácia da classificação de dois tipos de doenças, cercóspora e ferrugem. Para efeitos de comparação foi utilizada uma rede de aprendizado profundo, convolucional, para a mesma finalidade de classificação, porém, sem a obtenção prévia dos 
atributos de textura. Os resultados mostraram uma ligeira vantagem para o segundo método. Pagudpud [9] descreveu sobre uma pesquisa de exploração do uso de smartphones em comunidades de lavouras cafeeiras, nas Filipinas.

Quanto às técnicas de segmentação de imagens o algoritmo JSEG [10] tem sido muito explorada no processamento de imagens aplicado à agricultura. Lulio [11] utilizou o algoritmo JSEG para a segmentação de imagens de laranjais e classificação dos segmentos utilizando perceptron multicamadas. Diversos outros trabalhos fazendo uso do algoritmo JSEG também tem sido publicados, como o de Fambrini [12], que fez a aplicação desse algoritmo implementado para a finalidade de identificação de elementos da rede elétrica. O Algoritmo JSEG é multiescala, ou seja, a segmentação é obtida em 4 escalas, numeradas de 1 a 4 , sendo a escala 1, correspondente a segmentos de menor dimensão, e a escala 4, de maior dimensão. Não obstante, nos trabalhos encontrados na literatura não são descritas as vantagens de utilização de cada uma dessas escalas. Propõese neste trabalho analisar a segmentação das imagens do banco de dados disponível RoCoLe, utilizando o algoritmo JSEG, e analisar os resultados utilizando cálculo de histogramas e agrupamento dos segmentos nas diferentes escalas, fazendo uma comparação entre elas.

O restante do texto está subdividido nas seguintes seções: na Seção II é feita uma síntese sobre o algoritmo de segmentação JSEG; e a técnica de agrupamento utilizada; na Seção III, é apresentada a proposta de pesquisa e metodologia; na Seção IV são apresentados os resultados obtidos; e na Seção V, as conclusões e trabalhos futuros.

\section{Algoritmos: JSEg E AGRUPAMENTO}

Nesta seção, descreve-se inicialmente o algoritmo JSEG [10], seguido do algoritmo de agrupamento utilizado no trabalho.

\section{A. Algoritmo JSEG}

$\mathrm{O}$ algoritmo de segmentação baseado em imagens J, denotado JSEG, foi proposto por Deng Y. e Manjunath, B.S. [10]. É um método de segmentação baseada em textura de cores, que consiste em duas etapas independentes: 1) quantização de cores e 2) segmentação espacial, como ilustrado pelo diagrama da Figura 1 (a). Na primeira etapa, as cores da imagem são quantizadas em certo número de classes, e cada pixel recebe um rótulo correspondente à classe atribuída. Essa imagem resultante de rótulos de classes é denotada mapa de classes. Na segunda etapa Figura 1 (b), é realizada a segmentação espacial, na qual é inicialmente obtida uma imagem denotada $\mathrm{J}$, utilizando-se de janelas locais aplicadas a cada posição do mapa de classes. Essa imagem J possibilita a localização de posições centrais e de contorno dos segmentos a serem obtidos. Após a obtenção da imagem $\mathbf{J}$ é realizado o crescimento de regiões a partir das posições centrais. $\mathrm{O}$ tamanho das janelas locais determina $\mathrm{o}$ número máximo de pixels nos segmentos obtidos. Assim, para cada tamanho de janela tem-se a segmentação numa escala diferente. Na escala 1 , a janela é de 9x9, na escala 2 , de $17 \times 17$, na escala $3,33 \times 33$, e na escala $4,65 \times 65$. O tamanho típico de um segmento varia de acordo com a escala, sendo de $64 \times 64,128 \times 128,256 \times 256$ e 512x512, para as escalas 1,2 ,
3 e 4, respectivamente. A seguir, é descrita a definição de $J$, importante para o entendimento do algoritmo JSEG.

Seja $Z$ o conjunto de todos os $N$ pontos de dados num mapa de classes, entendendo pontos de dados como sendo as posições no espaço bidimensional $(x, y)$. Seja $z=(x, y), z \in Z$, e $m$ a média. Supõe-se que $Z$ é classificada em $C$ classes, $Z_{i}, i=$ $1, \ldots, C$. Seja $m_{j}$ a média dos $N_{j}$ pontos de dados de uma classe $Z_{j}$. Nota-se que como os pontos de dados representam as posições dos dados, a sua média tem o significado da posição central dos elementos que formam a classe. Sejam $S_{T}=$ $\sum_{z \in Z}\|z-m\|^{2}$ e $S_{w}=\sum_{i=1}^{C} S_{i}=\sum_{i=1}^{C} \sum_{z \in Z_{i}}\left\|z-m_{i}\right\|^{2} . S_{T}$ é a variância total dos pontos de $Z$ e $S_{W}$ é a soma das variâncias dos pontos pertencentes à mesma classe. Definimos $J$ pela equação (1):

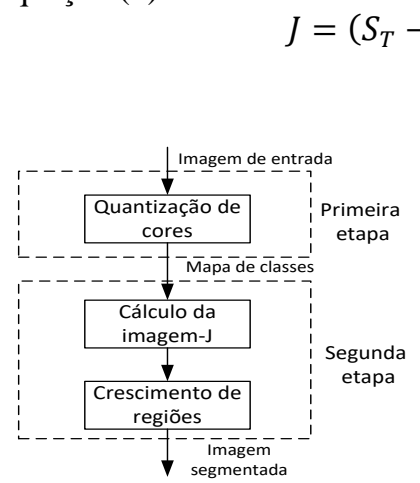

(a)
Figura 1. Diagrama de fluxo do algoritmo JSEG

Quando uma imagem tem várias regiões de cores homogêneas, as classes de cores são mais separadas uma da outra e o valor de $J$ é grande. Se as classes de cores são uniformemente distribuídas sobre uma imagem inteira, o valor de $J$ tende a ser pequeno. Seja agora, calcular $J$ sobre cada região segmentada ao invés do mapa de classes inteiro e definir o $\bar{J}$ pela equação (2):

$$
\bar{J}=\frac{1}{N} \sum_{k} M_{k} J_{k}
$$

onde $J_{k}$ é o $J$ calculado sobre a região $k, M_{k}$ é o número de pontos da região $k, N$ é o número total de pontos no mapa de classes, e a somatória é sobre todas as regiões do mapa de classes. A ideia do JSEG é fazer com que $\bar{J}$ seja o menor possível, sobre todas as formas de segmentação da imagem dado o número de regiões. Para um número fixo de regiões, uma melhor segmentação tende a ter um menor valor de $\bar{J}$. Se a segmentação é boa, cada região segmentada contém poucos rótulos de classes de cor distribuídos uniformemente e o valor $J$ resultante para aquela região é pequeno. Portanto, $\bar{J}$ também é pequeno, sendo que o valor mínimo de $\bar{J}$ é 0 . A minimização global de $\bar{J}$ para a imagem inteira não é prática, porém, observa-se que $J$, se aplicada a uma área local do mapa de classes, é também um bom indicador de se aquela área está dentro de uma região ou perto dos contornos. Podemos construir uma imagem cujos valores de pixels correspondem a $J$ calculados sobre pequenas janelas centradas nos pixels. Por conveniência, temos as imagens $J$ e os valores de pixels correspondentes como valor $J$ locais. Quanto maior o valor de $J$ local, mais provável que o pixel correspondente esteja perto de uma região de contorno. A imagem $J$ é como um mapa geográfico 3D contendo elevações e vales, que representam 
as regiões de contorno e interior, dos segmentos, respectivamente.

\section{B. Algoritmo de Agrupamento baseado na relação binária e de equivalência.}

Ao utilizar JSEG na segmentação de imagens, tem-se uma quantidade de segmentos, em quatro escalas. Para analisar o resultado, propõe-se realizar agrupamento de segmentos baseados na distância Euclidiana entre os seus histogramas normalizados, sendo pertencentes a um grupo, os segmentos com distâncias menores ou iguais a uma certa tolerância $\epsilon$. Essa técnica de agrupamento é baseada na teoria de Near Sets proposta por Peters [13][14], e justifica-se a sua utilização devido à definição clara dos limites entre os grupos. Nessa proposta os grupos podem ter sobreposições em relação aos outros grupos, e é baseado na relação binária e de equivalência, definidos a seguir. Uma relação binária do conjunto A no conjunto $\mathrm{B}$ é um subconjunto do produto cartesiano A x B. Para indicar que um par $\langle\mathrm{x}, \mathrm{y}\rangle$ pertence à relação $R$, escreve-se $x R y$. A relação $R$ no conjunto A é de equivalência se satisfizer às propriedades seguintes:

1. Reflexiva: se $x R x$ para todo $x \in A$;

2. Simétrica: se $x R y$ para $x, y \in A \rightarrow y R x$;

3. Transitiva: se $x R y$ e $y R z$ para $x, y, z \in A \rightarrow x R z$.

O Algoritmo de Agrupamento, descrito a seguir, considera que os grupos devem conter elementos que satisfaçam a relação binária e de equivalência.

\section{Algoritmo de Agrupamento: \\ Inicialização: \\ Para cada elemento $\mathrm{x}_{\mathrm{i}}, i=1, \ldots n$, obter o conjunto de vizinhos $V_{i}$, sendo um vizinho um elemento $\mathrm{x}_{\mathrm{j}}$ com a distância menor ou igual à tolerância $€$ de $\mathrm{x}_{\mathrm{i}}$. Criar também um conjunto auxiliar $A V_{i}$, inicialmente vazio.}

\section{Iteração:}

Repetir para todos $x_{i}, i=1, \ldots n$, os passos:

1) criar um grupo $G_{i}$, incluindo inicialmente o próprio elemento $\mathrm{x}_{\mathrm{i}}$ como pertencente à classe;

2) escolher um dos vizinhos dentro do conjunto $V_{i}$, com a menor distância em relação a $\mathrm{x}_{i}$; se não existir vizinhos no conjunto $V_{i}$, ir para o passo 6 .

3) Incorporar o vizinho escolhido ao grupo $G_{i}$, e calcular a distância entre o vizinho escolhido e os vizinhos remanescentes de $V_{i}$; Se não existirem vizinhos remanescentes ir para o passo 6 ;

4) dentre os vizinhos remanescentes de $V_{i}$, os que tiverem distância maior que $\epsilon$ para $o$ vizinho escolhido devem ser excluídos de $V_{i}$ e inseridos no conjunto $A V_{i}$;

5) ir para o passo 2;

6) caso o conjunto $A V_{i}$ não seja vazio, transferir todos os seus elementos para um novo conjunto de vizinhos $V_{i}$, esvaziar $A V_{i}$, e repetir os passos a partir do 1 , até que todos os vizinhos de $\mathrm{x}_{\mathrm{i}}$ estejam incluídos num dos grupos.
Saída: para cada elemento $\mathrm{x}_{\mathrm{i}}$ um conjunto de grupos é obtido como saída. Os grupos que forem contidos em outros grupos devem ser desprezados.

\section{Proposta de Pesouisa e metodologia}

A pesquisa consiste em segmentar um conjunto de imagens do banco de dados RoCoLe, utilizando o algoritmo JSEG, cujo resultado consiste em 4 escalas de segmentos. Como o banco de dados contém imagens de cafeeiros com doença de ferrugem, a proposta é de realizar o estudo dos segmentos obtidos nas diferentes escalas, para inferir sobre a escala mais adequada para a detecção dessas doenças. Para tanto é realizado um estudo de agrupamentos dos segmentos obtidos em cada escala, utilizando o algoritmo descrito na Seção IIB.

Propõe-se utilizar 50 imagens de 380x640 pixels para segmentação. Para cada segmento de imagem obtida, calcular o histograma normalizado de cores, para utilização no algoritmo de agrupamento. O algoritmo de agrupamento é aplicado para um conjunto de trinta segmentos considerados típicos para cada escala. Esse número de segmentos foi adotado por ter sido considerado suficiente para o estudo proposto. Após a aplicação do algoritmo de agrupamento para cada escala, uma análise comparativa dos resultados da segmentação é realizada, em função das características dos grupos obtidos de segmentos. A Figura 2 mostra um diagrama de blocos da sequência de experimentos proposta.

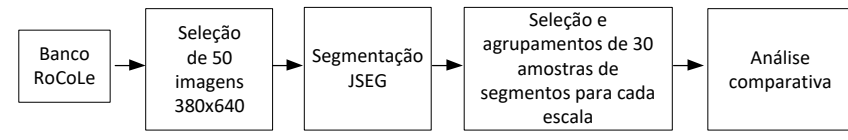

Figura 2. Diagrama de blocos da sequência de experimentos proposta.

As imagens extraídas do banco de dados RoCoLe, em 380x640 pixels são exemplificados na Figura 3, onde além das folhas são contidos fundos não foliares. Um exemplo de segmentação utilizando JSEG é mostrado na Figura 4, sendo (a) escala 1 com 273 segmentos, (b) escala 2, 108 segmentos (c ) escala 3, 29 segmentos e (d) escala 4, 6 segmentos, onde cada segmento é contornado por linhas brancas.
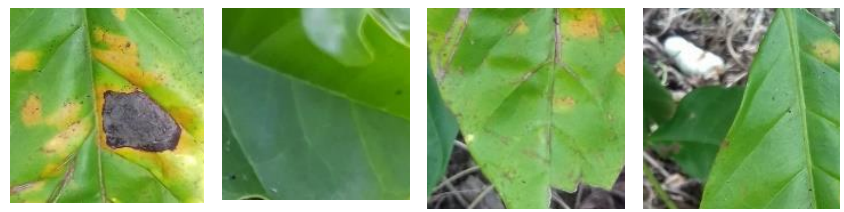

Figura 3. amostras de imagens extraídas do banco de dados RoCoLe.

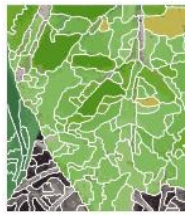

(a)

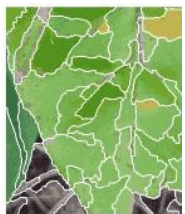

(b)

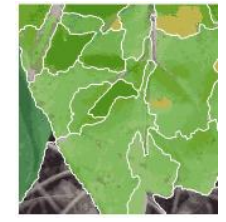

(c)

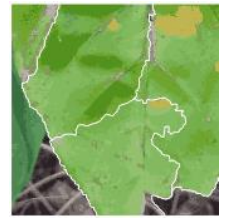

(d)
Figura 4. Exemplo de segmentação:

(a) escala 1, 273 segmentos (b) escala 2, 108 segmentos

(c) escala 3, 29 segmentos e (d) escala 4, 6 segmentos. 


\section{RESULTADOS E DISCUSSÕES}

Os experimentos foram realizados conforme a metodologia explicitada. Os resultados de segmentação, seleção de segmentos típicos para cada escala e os agrupamentos são descritos na ordem crescente de escala.

\section{A. Escala 1}

A Figura 5 mostra os segmentos típicos selecionados para a escala 1, em que a janela de segmentação foi de dimensão 9x9. A Figura 6 ilustra um dos segmentos, de número 23, e o seu respectivo histograma. Para efeitos de agrupamento, a numeração dos segmentos ocorre em duas linhas, iniciandose no canto esquerdo superior, com o número 1, terminando no canto direito inferior, com o número 30. Para o agrupamento dos segmentos, foi utilizada a tolerância de $\epsilon=$ 0,1 . Os grupos formados para esses segmentos estão mostrados na Tabela 1 e na Figura 7. Nota-se que foram obtidos 8 grupos de dois segmentos. Os grupos individuais de apenas um elemento não estão listados na tabela.

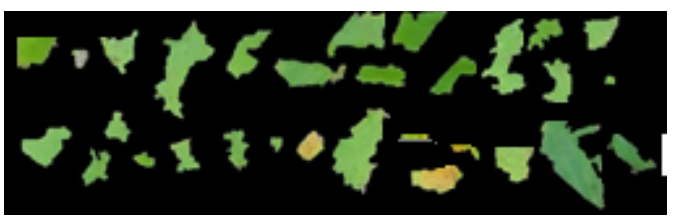

Figura 5 Segmentos típicos selecionados da escala 1.

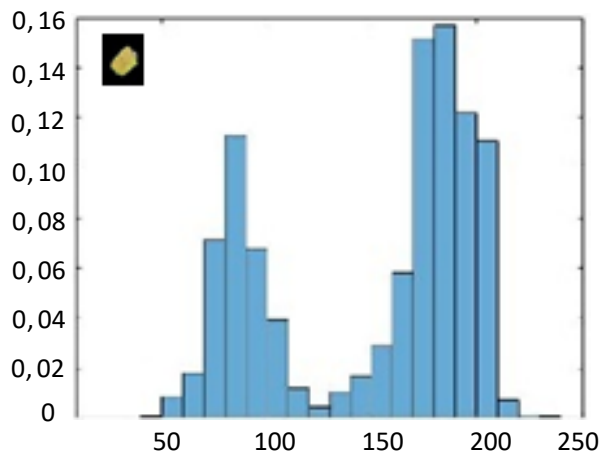

Figura 6 Ilustração de um histograma do segmento de número 23. No canto superior está mostrado o segmento.

\begin{tabular}{|c|c|c|c|}
\hline Tabela 1. Grupos para $\epsilon=0.1$ na escala 1 \\
\hline $\mathrm{G} 1=\{1,19\}$ & $\mathrm{G} 2=\{3,5\}$ & $\mathrm{G} 3=\{3,13\}$ & $\mathrm{G} 4=\{9,10\}$ \\
\hline
\end{tabular}

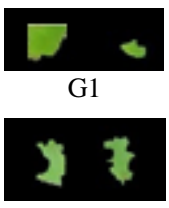

G5

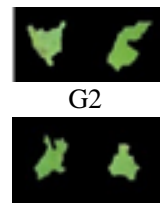

G6

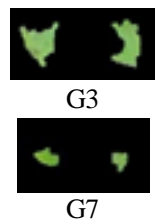

G7

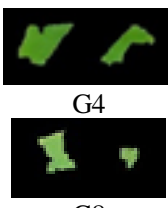

G8
Figura 7. Agrupamentos obtidos na escala 1, para $\epsilon=0.1$.

\section{B. Escala 2}

A Figura 8 refere-se aos segmentos típicos selecionados para a escala 2, em que a janela de segmentação foi de dimensão 17x17. A Figura 9 ilustra um dos segmentos, de número 28, e o seu respectivo histograma. A numeração dos segmentos ocorre da mesma forma que para a escala 1. Para o agrupamento, foi também utilizada a tolerância $\epsilon=0,1$. Os grupos formados para esses segmentos estão mostrados na Tabela 2 e na Figura 10. Nota-se que foram obtidos 13 grupos de dois ou mais segmentos, sendo que os grupos individuais não estão listados na tabela.

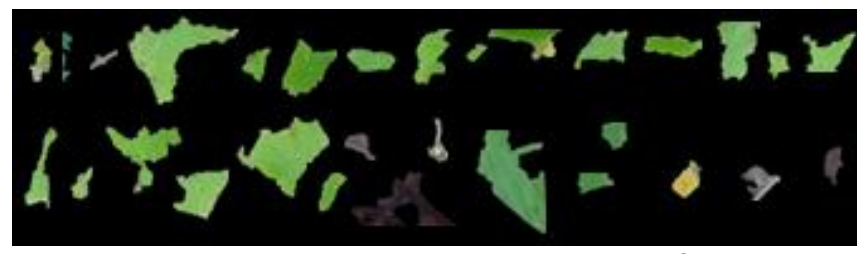

Figura 8 Segmentos típicos selecionados da escala 2

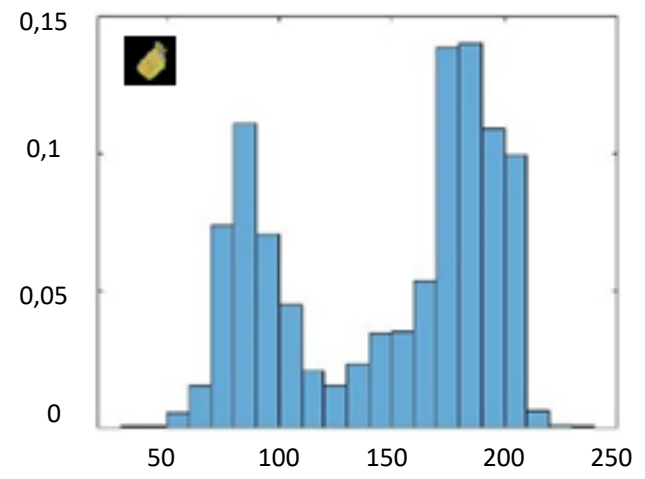

Figura 9 Ilustração de um histograma do segmento de número 28. No canto superior está mostrado o segmento.

Tabela 2. Grupos para $\epsilon=0.1$ para a escala 2

\begin{tabular}{|c|c|c|c|}
\hline $\mathrm{G} 1=\{1,3\}$ & $\mathrm{G} 2=\{1,28\}$ & $\mathrm{G} 3=\{3,29\}$ & $\mathrm{G} 4=\{4,16,18\}$ \\
\hline $\mathrm{G} 5=\{5,8\}$ & $\mathrm{G} 6=\{5,20\}$ & $\mathrm{G} 7=\{7,19\}$ & $\mathrm{G} 8=\{10,29\}$ \\
\hline $\mathrm{G} 9=\{16,20\}$ & $\mathrm{G} 10=\{16,29\}$ & $\mathrm{G} 11=\{17,20\}$ & $\mathrm{G} 12=\{20,23\}$ \\
\hline $\mathrm{G} 13=\{22,24,29\}$ & & & \\
\hline
\end{tabular}
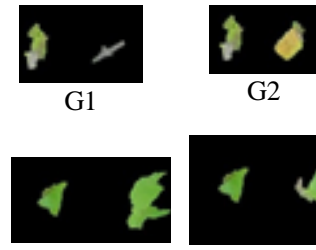

G5

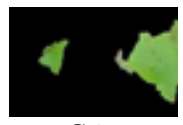

G6

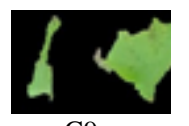

G9

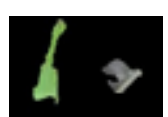

G10

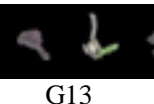

G13

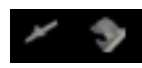

G3

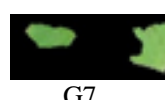

G7

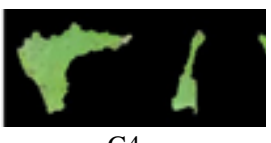

G4

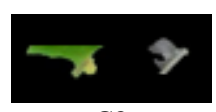

G8

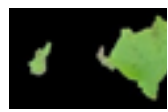

G11

G12

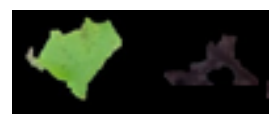

\section{Escala 3}

A Figura 11 refere-se aos segmentos típicos selecionados para a escala 3 , em que a janela de segmentação foi de dimensão 33x33. A Figura 12 referese a uma ilustração de um histograma, juntamente com o respectivo segmento no canto superior esquerdo. A numeração dos segmentos ocorre, em três linhas, sendo 
dez segmentos por linha. Para o agrupamento, foi utilizada a tolerância $\epsilon=0,1$. Os grupos formados para esses segmentos estão mostrados na Tabela 3 e na Figura 13. Foram obtidos 8 grupos de dois ou mais segmentos, sendo que os grupos individuais não estão listados na tabela.

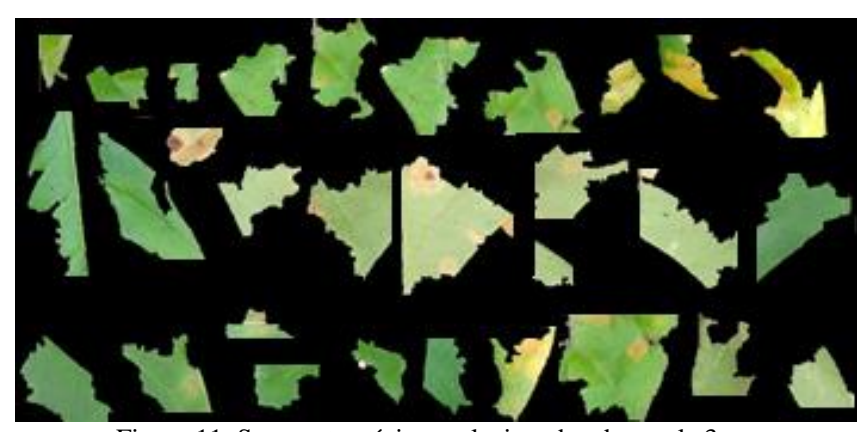

Figura 11. Segmentos típicos selecionados da escala 3.

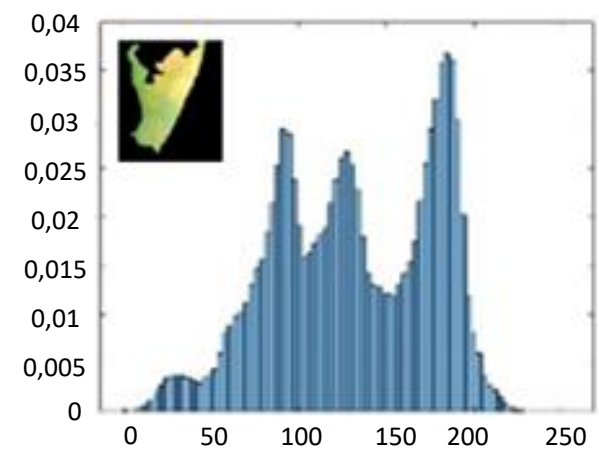

Figura12. Ilustração de um histograma do segmento de número 23. No canto superior está mostrado o segmento.

Tabela 3. Grupos com para $\epsilon=0.1$ para a escala 3

\begin{tabular}{|c|c|c|c|}
\hline $\mathrm{G} 1=\{1,8,13\}$ & $\mathrm{G} 2=\{2,4,7\}$ & $\mathrm{G} 3=\{4,5,6\}$ & $\mathrm{G} 4=\{1,9,27\}$ \\
\hline $\mathrm{G} 5=\{1,5,8\}$ & $\mathrm{G} 6=\{15,29,30\}$ & $\mathrm{G} 7=\{24,25\}$ & $\mathrm{G} 8=\{15,28,29]$ \\
\hline
\end{tabular}

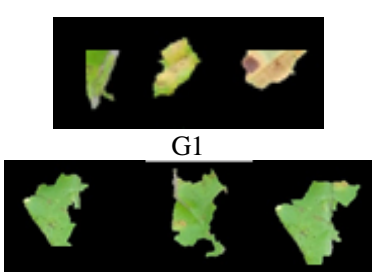

G3

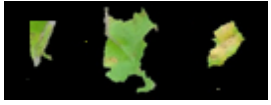

G5

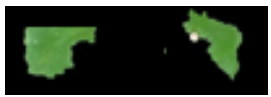

G7

Figura 13. Agrupamentos obtidos com a escala 3

\section{Escala 4}

A Figura 14 refere-se aos segmentos típicos selecionados para a escala 4 , em que a janela de segmentação foi de dimensão $65 \times 65$. A numeração dos segmentos ocorre da mesma forma que no caso anterior. A Figura 15 refere-se a uma ilustração de um histograma típico na escala 4, mais precisamente do segmento 14. Para o agrupamento, foi também utilizada a tolerância $\epsilon=0,1$. Os grupos formados para esses segmentos estão mostrados na Tabela 4 e alguns exemplos na Figura 16. Foram obtidos 21 grupos de dois ou mais segmentos, sendo que os grupos individuais não estão listados na tabela.

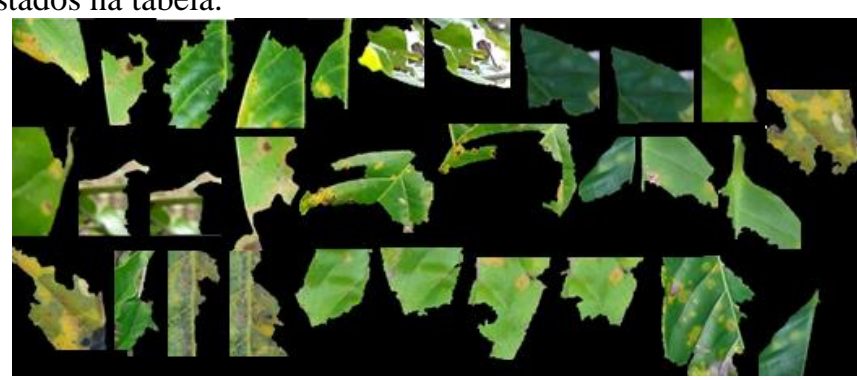

Figura 14. Segmentos típicos selecionados da escala 4.

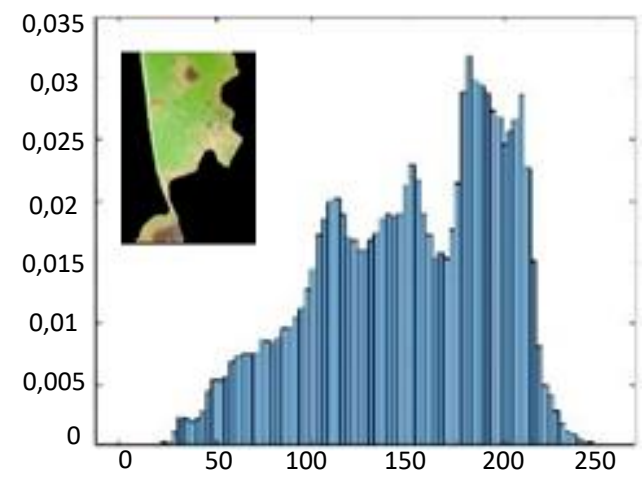

Figura15. Ilustração de um histograma do segmento de número 23. No canto superior está mostrado o segmento.

\begin{tabular}{|c|c|}
\hline $\mathrm{G} 1=\{1,15,16,17\}$ & $\mathrm{G} 2=\{1,2,12,13,15,25,26,27\}$ \\
\hline $\mathrm{G} 3=\{1,2,12,13,16,24,25,26,27\}$ & $\mathrm{G} 4=\{1,12,13,17,23,25,26,27\}$ \\
\hline $\mathrm{G} 5=\{1,2,4,23,24,27,30\}$ & $\mathrm{G} 6=\{1,2,12,13,14,23,24,25,26,27,30$ \\
\hline $\mathrm{G} 7=\{2,15,16,20,21\}$ & $\mathrm{G} 8=\{3,4,5,15,16,22\}$ \\
\hline $\mathrm{G} 9=\{3,4,15,16,17,22,29,30\}$ & $\mathrm{G} 10=\{3,12,13,15,16,17,25,26\}$ \\
\hline G11 $=\{6,7\}$ & $\mathrm{G} 12=\{8,9\}$ \\
\hline G13 $=\{10,11\}$ & $\mathrm{G} 14=\{10,12,13,15,16\}$ \\
\hline $\mathrm{G} 15=\{12,15,16,20,21\}$ & G16 $=\{12,28\}$ \\
\hline $\mathrm{G} 17=\{15,28\}$ & $\mathrm{G} 18=\{16,20,21\}$ \\
\hline $\mathrm{G} 19=\{18,19\}$ & $\mathrm{G} 20=\{20,21,30\}$ \\
\hline $\mathrm{G} 21=\{28,30\}$ & \\
\hline
\end{tabular}

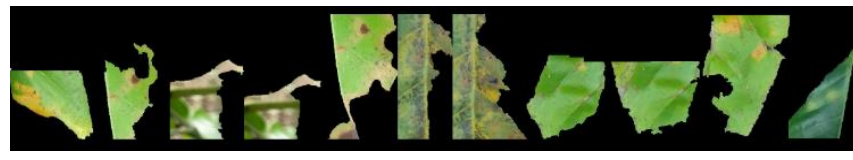

(a)

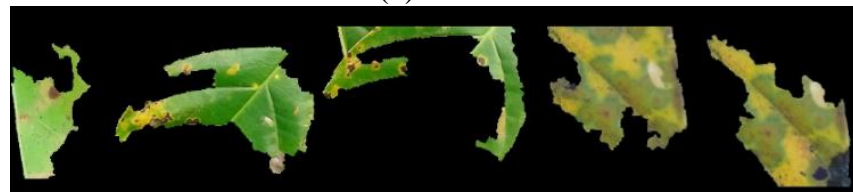

(b)

Figura 16. (a) agrupamento G6, (b) agrupamento G7.

\section{E. Discussões}

Nota-se que os segmentos ao aumentar a escala, são em número menor e maiores para uma mesma imagem. Na escala 
1, os segmentos são visivelmente pequenos em área e mais homogêneos, e na escala 4, os segmentos contêm mais pixels e são mais heterogêneos. Os histogramas mostrados, nas Figuras 6, 9, 12 e 15, respectivamente, das escalas 1, 2, 3 e 4, contêm manchas correspondentes à ferrugem. Pelos histogramas das Figuras 6 e 9, é possível ver que essa mancha contém dois módulos de cores, com picos nas intensidades de cores 80 e 180, respectivamente. Já na ilustração das Figuras 12 e 15, os histogramas contêm três módulos de cores. Isso mostra que nas escalas 3 e 4, os segmentos são mais heterogêneos, e isso é visível pois nota-se a presença da parte esverdeada. Em termos de utilização da segmentação na detecção de doenças, quanto mais homogêneo seja o segmento, mais fácil seria a detecção. Isso significa que a segmentação nas escalas 1 e 2, poderiam ser usadas para essa finalidade. Nota-se que na escala 1 os elementos agrupados parecem muito mais próximos que os grupos na escala 2 e assim sucessivamente, apesar de ter utilizado a mesma tolerância $\epsilon=0,1$.

\section{CONCLUSÕES E TRABALHOS FUTUROS}

Este trabalho teve como finalidade o desenvolvimento de um sistema que faz a segmentação das imagens de cafeeiros utilizando o algoritmo JSEG, e posterior agrupamento dos segmentos. Foi possível obter resultados em quatro escalas de segmentação, em função do tamanho das janelas utilizadas que variam de 9x9 a 65x65. Nota-se que os segmentos na escala 1, a dimensão em pixels é menor, e as cores são mais homogêneas, enquanto nas escalas maiores, a dimensão é maior, e as cores são mais heterogêneas. Para efeitos de detecção de doenças nos cafeeiros, em que as partes lesadas aparecem em cores avermelhadas, o uso da segmentação em escalas 1 e 2 parece mais adequado. Como proposta de trabalhos futuros sugere-se o treinamento de redes neurais, em que as entradas sejam os valores dos histogramas normalizados obtidos a partir da segmentação JSEG. Para tanto, os segmentos contendo manchas de doenças, nas escalas 1 ou 2, devem ser selecionados como amostras de treinamento.

\section{AgradeCimentos}

Os autores agradecem ao Prof. Dr. Francisco Fambrini por gentilmente permitir o uso da versão do código JSEG neste trabalho, e à CAPES (Coordenação de Aperfeiçoamento de Pessoal de Nível Superior), Ministério da Educação, código de financiamento 001 .

\section{REFERÊNCIAS}

[1] C.A.Krohing, O.J.Rocha, F.J.Eutrópio, A.G.Silva, Uma avaliação do ataque da ferrugem do cafeeiro, Hemileia vastatrix Berk.\&Br., no sub-bosque da Reserva Biológica de Duas Bocas, Cariacica, Espírito Santo, Natureza on line, vol.8, n.2, pp.63-66, 2010.

[2] D.Suhartono, W.A.Derwin, L.Miranty, Y.Muhamad, Expert System in Detecting Coffee Plant Diseases, International Journal of Electrical Energy, vol. 1, n.3, pp.156-162, 2013.
[3] J.G.A. Barbedo, A Review on the Main Challenges in Automatic Plant Disease Identification Based on Visible Range Images, Biosystems Engineering, vol. 144, pp. 52-60, 2016.

[4] V. Metre, J. Ghorpade, An Overview of the Research on Texture Based Plant Leaf Classification, IJCSNInternational Journal of Computer Science and Network, vol.2, n.3, pp. 1-12, 2013.

[5] A. D. Mengistu, D.M.Alemayehu, S.G.Mengistu, Ethiopian Coffee Plant Diseases Recognition Based on Imaging and Machine Learning Techiniques, International Journal of Database Theory and Applications, vol.9, n.4, pp.79-88, 2016.

[6] A.S.A.Mettleq, S.Abu-Naser, A Rule Based System for the Diagnosis of Coffee Diseases, International Journal of Academic Information Systems Research, vol. 3, pp. 1-8, 2019.

[7] J. Parraga-Alava, K.Cusme, A. Loor, E. Santander, RoCoLe: A robusta coffee leaf images dataset for evaluation of machine learning based methods in plant diseases recognition, Data in Brief, vol.25 pp. 1-5,2019.

[8] L.X.B.Sorte, C.T.Ferraz, F.Fambrini, R.R.Goulart, J.H.Saito, Coffee Leaf Disease Recognition Based on Deep Learning and Texture Attributes, Procedia Computer Science, vo.159, pp.135-144, 2019.

[9] M.V.Pagudpud, Exploring the Smartphone Manipulation Skills in a Coffee Farming Community Using Clustering Algorithm, ICMSSP: Proceedings of the 2009th 4th International Conference on Multimedia Systems and Signal Processing, pp.52-56, maio de 2019.

[10] Y.Deng, B.S. Manjunath, Unsupervised Segmentation of Color-Texture Regions in Images and Video, IEEE Transactions on Pattern Analysis and Machine Intelligence, vol.23, n.8, pp. 800-810, 2001.

[11] L.C.Lulio, M.L.Tronco, A.J.V. Porto, JSEG-based image segmentation in computer vision for agricultural mobile robot navigation, IEEE International Symposium on Computational Intelligence in Robotics and Automation (CIRA), pp. 1-6, 15-18 Dezembro, 2009.

[12] F.Fambrini, Y. Iano, D.G.Caetano, A.A.D. Rodriguez, C. Moya, E. Carrara, A. Rangel, F.C. Cabelo, J.V. Zubem, L.M.D.V. Cura, J.B. Destro-Filho, J.R. Campos, J.H. Saito, GPU Cuda JSEG Segmentation Algorithm associated with Deep Learning Classifier for Electrical Network Images Identification, Procedia Computer Science, vol. 126, pp. 557-565, 2018.

[13] C.J. Henry, Near Sets: Theory and Applications, Academic Doctoral Thesis, University of Manitoba, Winnipeg, Manitoba R3T 5V6, Canada, 2010.

14] J.F. Peters, Near Sets, General Theory About Nearness of Objects, Applied Mathematical Sciences, vol.1, n.53, pp.2609-2629, 2007. 